\title{
Crystal Structure of a Fluoroquinolone Antibiotic, Enoxacin
}

\author{
Il Yoon,* Ki-Min ParK,* Ki-Min BARK,** Min Suk Yang,*** and Shim Sung LeE*广 \\ *Department of Chemistry and Research Institute of Natural Sciences, Gyeongsang National University, \\ Chinju 660-701, S. Korea \\ **Department of Chemistry Education, Gyeongsang National University, Chinju 660-701, S. Korea \\ ***Department of Agricultural Chemistry, Division of Applied Life Science, Gyeongsang National University, \\ Chinju 660-701, S. Korea
}

\begin{abstract}
Crystals of Enoxacin, one of commercial fluoroquinolone antibiotics, were grown from acetonitrile/methanol and the crystal structure was determined. Results show that the unit cell contains two crystallographically independent molecules (A and B), three water and one methanol molecules. The carboxylic group is ionized and the N4 atom from the piperazine ring is protonated, resulting a zwitter ionic structure. The interlayer $\pi$ - $\pi$ interactions and the intermolecular hydrogen bonds stabilize the crystal packing.
\end{abstract}

(Received October 15, 2002; Accepted April 24, 2003; Published on Web July 18, 2003)

Fluoroquinolones are very useful antibiotics, which are increasingly being used for the treatment of many infections. Enoxacin (1-ethyl-6-fluoro-1,4-dihydro-4-oxo-7-(1-piperazinyl)-1,8-naphthyridine-3-carboxylic acid) (Fig. 1) is one of these agents, and used to treat certain infections caused by bacteria, such as gonorrhea and urinary tract infections.,3 In spite of a large amount of data on the activity of enoxacin in biology and chemotherapy, its crystal structure has not been available. Herein, we describe the structure of this molecule based on an $\mathrm{X}$-ray crystal analysis.

Enoxacin was purchased from Sigma Co. and used without further purification. Colorless crystals suitable for X-ray crystallography were grown by slow evaporation from acetonitrile/methanol $(9: 1)$ at room temperature. The crystal and experimental data are given in Table 1. The structure was solved by a direct method. $\mathrm{H}$ atoms bonded to the $\mathrm{N}$ atom of the piperazine ring were found from difference Fourier maps and then refined freely. $\mathrm{H}$ atoms of the water molecules were not included. Other $\mathrm{H}$ atoms were included in the calculated positions with isotropic thermal parameters 1.2-times those of the attached atoms. The atomic coordinates and selected geometric parameters are presented in Tables 2 and 3, respectively. The molecular structure and the atomic numbering are given in Fig. 2 .

The unit cell contains two crystallographically independent

Fig. 1 Chemical structure.

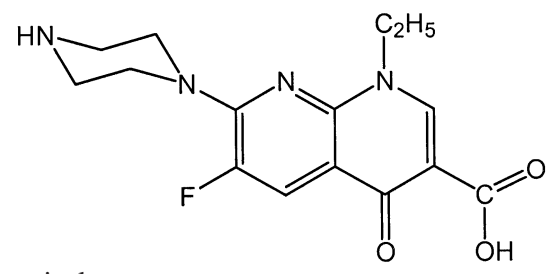

\footnotetext{
$\doteqdot$ To whom correspondence should be addressed.

E-mail: sslee@ nongae.gsnu.ac.kr
}

molecules, A and B, together with three water and one methanol molecules. Molecules A and B show no significance difference in bond lengths and angles. The carboxylic group is in the ionized form, showing the typical bond distances of carboxylic anion in the range of 1.248(4) - 1.263(4) $\AA$, and the N4 atom of the piperazine ring is protonated, resulting a zwitter ionic structure in both molecules. There was no residual density near carboxylic $\mathrm{O}$ atoms, which can be assigned by $\mathrm{H}$ atoms. The

Table 1 Crystal and experimental data

Formula: $\mathrm{C}_{31} \mathrm{H}_{44} \mathrm{~F}_{2} \mathrm{~N}_{8} \mathrm{O}_{10}$

Formula weight $=726.74$

Temperature: $100 \mathrm{~K}$

Crystal size: $0.40 \times 0.20 \times 0.20 \mathrm{~mm}$

Crystal color: colorless

Crystal description: plate

Crystal system: monoclinic

Space group: $P 2_{1} / c \quad Z=4$

$a=8.4053(6) \AA$

$b=22.1236(16) \AA$

$c=17.1548(12) \AA$

$\beta=100.903(1)^{\circ}$

$V=3132.4(4) \AA^{3}$

$D_{\mathrm{x}}=1.541 \mathrm{~g} / \mathrm{cm}^{3}$

Radiation: 0.71073 (Mo K $\left.{ }_{\alpha}\right)$

$2 \theta_{\max }=56.56^{\circ}$

$R=0.0683$

$w R_{2}=0.2242$

Goodness-of-fit $=1.059$

$(\Delta / \sigma)_{\max }$ final cycle: 0.000

$(\Delta \rho)_{\max }=0.952 \mathrm{e}^{-3}$

$(\Delta \rho)_{\min }=-0.930 \mathrm{e}^{-3}$

No. of reflections used $=7395[>2 \sigma(I)]$

No. of parameters $=461$

Diffractometer: Bruker SMART CCD system

Monochromater: graphite

Structure determination: SHELXTL

Refinement: full-matrix least-squares 
Table 2 Atomic coordinates $\left(\times 10^{4}\right)$ and equivalent isotropic displacement parameters $\left(\AA^{2} \times 10^{3}\right)$

\begin{tabular}{|c|c|c|c|c|}
\hline Atom & $x$ & $y$ & $z$ & $U(\mathrm{eq})$ \\
\hline $\mathrm{F}(1 \mathrm{~A})$ & $2144(2)$ & $7410(1)$ & $6097(1)$ & $16(1)$ \\
\hline $\mathrm{O}(1 \mathrm{~A})$ & $4005(3)$ & $9103(1)$ & $4559(1)$ & $20(1)$ \\
\hline $\mathrm{O}(2 \mathrm{~A})$ & $5291(3)$ & $9661(1)$ & $3337(1)$ & $24(1)$ \\
\hline $\mathrm{O}(3 \mathrm{~A})$ & 6991(3) & $9088(1)$ & $2819(1)$ & $25(1)$ \\
\hline $\mathrm{N}(1 \mathrm{~A})$ & $5439(3)$ & $7515(1)$ & $3661(1)$ & 13(1) \\
\hline $\mathrm{N}(2 \mathrm{~A})$ & $4350(3)$ & $6952(1)$ & $4579(1)$ & $12(1)$ \\
\hline $\mathrm{N}(3 \mathrm{~A})$ & $3256(3)$ & $6348(1)$ & $5450(1)$ & 14(1) \\
\hline $\mathrm{N}(4 \mathrm{~A})$ & $2705(3)$ & $5148(1)$ & $5968(2)$ & $18(1)$ \\
\hline $\mathrm{C}(1 \mathrm{~A})$ & $5931(3)$ & $9164(1)$ & $3239(2)$ & $15(1)$ \\
\hline$C(2 A)$ & $5798(3)$ & $8057(1)$ & $3370(2)$ & $14(1)$ \\
\hline$C(3 A)$ & $5394(3)$ & $8609(1)$ & $3636(2)$ & $14(1)$ \\
\hline $\mathrm{C}(4 \mathrm{~A})$ & 4499(3) & $8635(1)$ & $4280(2)$ & $15(1)$ \\
\hline $\mathrm{C}(5 \mathrm{~A})$ & $3367(3)$ & $8009(1)$ & $5253(2)$ & $13(1)$ \\
\hline $\mathrm{C}(6 \mathrm{~A})$ & $3055(3)$ & $7453(1)$ & $5524(2)$ & $12(1)$ \\
\hline $\mathrm{C}(7 \mathrm{~A})$ & $3586(3)$ & $6915(1)$ & $5193(2)$ & 13(1) \\
\hline $\mathrm{C}(8 \mathrm{~A})$ & $4629(3)$ & $7502(1)$ & $4297(2)$ & $12(1)$ \\
\hline$C(9 A)$ & $4201(3)$ & $8045(1)$ & $4612(2)$ & 13(1) \\
\hline $\mathrm{C}(10 \mathrm{~A})$ & $5836(3)$ & $6953(1)$ & $3267(2)$ & $15(1)$ \\
\hline $\mathrm{C}(11 \mathrm{~A})$ & $4594(4)$ & $6824(2)$ & $2526(2)$ & 24(1) \\
\hline $\mathrm{C}(12 \mathrm{~A})$ & $3434(4)$ & $6211(1)$ & $6304(2)$ & $16(1)$ \\
\hline $\mathrm{C}(13 \mathrm{~A})$ & $2330(4)$ & $5690(1)$ & $6420(2)$ & 19(1) \\
\hline $\mathrm{C}(14 \mathrm{~A})$ & 2617(4) & $5291(1)$ & $5113(2)$ & $16(1)$ \\
\hline$C(15 A)$ & $3700(4)$ & $5824(1)$ & $5009(2)$ & $16(1)$ \\
\hline $\mathrm{F}(1 \mathrm{~B})$ & $2795(2)$ & $2569(1)$ & $8614(1)$ & 23(1) \\
\hline $\mathrm{O}(1 \mathrm{~B})$ & 1149(3) & $891(1)$ & $10277(1)$ & 21(1) \\
\hline $\mathrm{O}(2 \mathrm{~B})$ & $306(3)$ & $394(1)$ & 11671(1) & $27(1)$ \\
\hline $\mathrm{O}(3 \mathrm{~B})$ & $-1673(3)$ & $930(1)$ & $12043(1)$ & 19(1) \\
\hline $\mathrm{N}(1 \mathrm{~B})$ & $-68(3)$ & $2509(1)$ & $11207(1)$ & $14(1)$ \\
\hline $\mathrm{N}(2 \mathrm{~B})$ & $864(3)$ & $3048(1)$ & $10229(1)$ & 14(1) \\
\hline $\mathrm{N}(3 \mathrm{~B})$ & $1840(3)$ & $3661(1)$ & $9342(1)$ & $16(1)$ \\
\hline N(4B) & 2592(3) & $4865(1)$ & $8912(2)$ & $19(1)$ \\
\hline $\mathrm{C}(1 \mathrm{~B})$ & $-518(4)$ & $871(1)$ & $11680(2)$ & $16(1)$ \\
\hline $\mathrm{C}(2 \mathrm{~B})$ & $-417(3)$ & 1972(1) & $11514(2)$ & 14(1) \\
\hline$C(3 B)$ & $-68(3)$ & $1415(1)$ & $11239(2)$ & $15(1)$ \\
\hline $\mathrm{C}(4 \mathrm{~B})$ & $726(3)$ & $1372(1)$ & $10560(2)$ & $14(1)$ \\
\hline$C(5 B)$ & 1719(3) & 1993(1) & $9529(2)$ & $15(1)$ \\
\hline $\mathrm{C}(6 \mathrm{~B})$ & 2001(3) & $2545(1)$ & $9239(2)$ & $16(1)$ \\
\hline $\mathrm{C}(7 \mathrm{~B})$ & $1548(3)$ & $3093(1)$ & $9589(2)$ & $15(1)$ \\
\hline $\mathrm{C}(8 \mathrm{~B})$ & $619(3)$ & $2504(1)$ & $10527(2)$ & 13(1) \\
\hline $\mathrm{C}(9 \mathrm{~B})$ & $989(3)$ & $1955(1)$ & 10201(2) & $14(1)$ \\
\hline $\mathrm{C}(10 \mathrm{~B})$ & $-319(3)$ & $3105(1)$ & $11565(2)$ & $15(1)$ \\
\hline $\mathrm{C}(11 \mathrm{~B})$ & $-1022(4)$ & $3064(1)$ & $12309(2)$ & 21(1) \\
\hline $\mathrm{C}(12 \mathrm{~B})$ & 1881(4) & $3815(1)$ & $8514(2)$ & $18(1)$ \\
\hline $\mathrm{C}(13 \mathrm{~B})$ & $3085(4)$ & $4325(1)$ & $8491(2)$ & $20(1)$ \\
\hline$C(14 B)$ & $2437(4)$ & $4716(1)$ & $9745(2)$ & 19(1) \\
\hline $\mathrm{C}(15 \mathrm{~B})$ & $1302(4)$ & $4183(1)$ & $9765(2)$ & $18(1)$ \\
\hline $\mathrm{O}(1 \mathrm{~W})$ & $-68(3)$ & $4611(1)$ & $2126(1)$ & $25(1)$ \\
\hline $\mathrm{O}(2 \mathrm{~W})$ & $2479(3)$ & $5677(1)$ & $1404(1)$ & $28(1)$ \\
\hline $\mathrm{O}(3 \mathrm{~W})$ & $2388(10)$ & $4281(4)$ & $3667(4)$ & $155(3)$ \\
\hline $\mathrm{O}(7)$ & $3920(5)$ & $5004(2)$ & $3380(2)$ & $62(1)$ \\
\hline $\mathrm{C}(31)$ & $4774(6)$ & $5357(2)$ & $3073(3)$ & $55(1)$ \\
\hline
\end{tabular}

$U_{\mathrm{eq}}=1 / 3 \Sigma_{i} \Sigma_{j} U_{i j} a_{i}^{*} a_{j}^{*}\left(\boldsymbol{a}_{i} \cdot \boldsymbol{a}_{j}\right)$.

largest difference Fourier peak $\left(0.95 \mathrm{e}^{-3}\right)$ is located at a short distance $(0.06 \AA)$ from $\mathrm{O} 3 \mathrm{~W}$ of the non-hydrated water molecule. The naphthyridone rings are almost planar with average deviations of $0.015(2) \AA$ for molecule A and $0.017(2) \AA$ for molecule B, respectively, from the least-squares planes defined by all non-hydrogen atoms. The molecules are stacked by $\pi-\pi$ interactions (average distance of two planes, $3.40(1) \AA$ ). The intermolecular hydrogen bonds including solvent molecules also stabilize the molecular packing (figure not shown). The hydrogen-bonding parameters are summarized in Table 4.

\section{Acknowledgements}

Support of the Korea Research Foundation (Project No. 2001-
Table 3 Selected bond lengths $(\AA)$ and angles $\left({ }^{\circ}\right)$

\begin{tabular}{llll}
\hline Molecule A & & Molecule B & \\
F(1A)-C(6A) & $1.359(3)$ & F(1B)-C(6B) & $1.366(3)$ \\
O(1A)-C(4A) & $1.243(3)$ & O(1B)-C(4B) & $1.248(3)$ \\
O(2A)-C(1A) & $1.248(4)$ & O(2B)-C(1B) & $1.263(4)$ \\
O(3A)-C(1A) & $1.259(4)$ & O(3B)-C(1B) & $1.255(4)$ \\
N(1A)-C(2A) & $1.354(3)$ & N(1B)-C(2B) & $1.354(4)$ \\
N(1A)-C(8A) & $1.393(3)$ & N(1B)-C(8B) & $1.396(4)$ \\
N(1A)-C(10A) & $1.484(3)$ & N(1B)-C(10B) & $1.486(3)$ \\
N(2A)-C(7A) & $1.336(4)$ & N(2B)-C(7B) & $1.337(4)$ \\
N(2A)-C(8A) & $1.346(3)$ & N(2B)-C(8B) & $1.338(3)$ \\
N(3A)-C(7A) & $1.375(3)$ & N(3B)-C(7B) & $1.364(4)$ \\
N(3A)-C(12A) & $1.476(3)$ & N(3B)-C(12B) & $1.468(4)$ \\
N(3A)-C(15A) & $1.471(3)$ & N(3B)-C(15B) & $1.478(4)$ \\
N(4A)-C(13A) & $1.494(4)$ & N(4B)-C(13B) & $1.495(4)$ \\
N(4A)-C(14A) & $1.489(4)$ & N(4B)-C(14B) & $1.497(4)$ \\
& & & \\
C(2A)-N(1A)-C(8A) & $118.9(2)$ & C(2B)-N(1B)-C(8B) & $118.2(2)$ \\
C(7A)-N(2A)-C(8A) & $118.6(2)$ & C(7B)-N(2B)-C(8B) & $120.1(2)$ \\
C(15A)-N(3A)-C(12A) & $111.2(2)$ & C(15B)-N(3B)-C(12B) & $111.0(2)$ \\
C(14A)-N(4A)-C(13A) & $111.3(2)$ & C(14B)-N(4B)-C(13B) & $111.5(2)$ \\
O(2A)-C(1A)-O(3A) & $124.3(3)$ & O(2B)-C(1B)-O(3B) & $124.8(3)$ \\
\hline
\end{tabular}
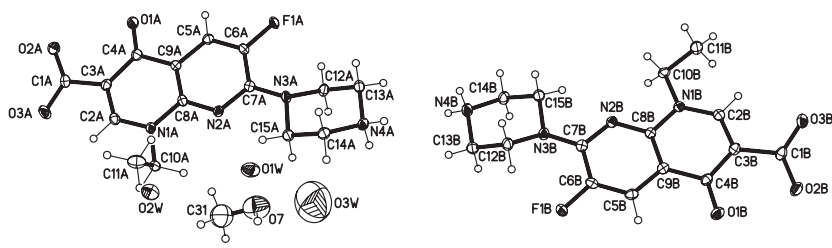

Fig. 2 ORTEP drawing of the title compound, representing heavy atoms as $30 \%$ probability ellipsoids and $\mathrm{H}$ atoms as spheres of arbitrary radius.

Table 4 Hydrogen-bonding geometry $\left(\AA,^{\circ}\right)$

\begin{tabular}{|c|c|c|c|c|}
\hline D-HA & D-H $(\AA)$ & $\mathrm{HA}(\AA)$ & $\mathrm{DA}(\AA)$ & D-HA $\left({ }^{\circ}\right)$ \\
\hline $\mathrm{N}(4 \mathrm{~A})-\mathrm{H}(4 \mathrm{~A}) \mathrm{O}(7)^{\mathrm{i}}$ & 0.900 & 1.998 & $2.870(5)$ & 161.6 \\
\hline $\mathrm{N}(4 \mathrm{~A})-\mathrm{H}(4 \mathrm{~B}) \mathrm{O}(1 \mathrm{~B})^{\mathrm{ii}}$ & 0.900 & 2.112 & $2.794(3)$ & 131.9 \\
\hline $\mathrm{N}(4 \mathrm{~A})-\mathrm{H}(4 \mathrm{~B}) \mathrm{O}(2 \mathrm{~B})^{\mathrm{ii}}$ & 0.900 & 2.046 & $2.806(3)$ & 141.5 \\
\hline $\mathrm{N}(4 \mathrm{~B})-\mathrm{H}(4 \mathrm{D}) \mathrm{O}(1 \mathrm{~A})^{\mathrm{iii}}$ & 0.900 & 1.989 & $2.714(3)$ & 136.5 \\
\hline $\mathrm{N}(4 \mathrm{~B})-\mathrm{H}(4 \mathrm{D}) \mathrm{O}(2 \mathrm{~A})^{\mathrm{iii}}$ & 0.900 & 2.118 & $2.840(3)$ & 136.7 \\
\hline $\mathrm{N}(4 \mathrm{~B})^{\mathrm{iv}}-\mathrm{H}(4 \mathrm{C}) \mathrm{O}(1 \mathrm{~W})$ & 0.900 & 1.885 & $2.755(4)$ & 161.8 \\
\hline $\mathrm{O}(7)-\mathrm{H}(7) \mathrm{O}(3 \mathrm{~W})$ & 0.960 & 1.844 & $2.731(3)$ & 96.1 \\
\hline $\mathrm{O}(7)-\mathrm{H}(7) \mathrm{O}(3 \mathrm{~A})^{v}$ & 0.960 & 1.935 & $2.817(3)$ & 170.0 \\
\hline $\mathrm{O}(7) \mathrm{O}(3 \mathrm{~B})^{\mathrm{vi}}$ & & & $2.907(3)$ & \\
\hline $\mathrm{O}(3 \mathrm{~A})^{\mathrm{v}} \mathrm{O}(1 \mathrm{~W})$ & & & $2.168(9)$ & \\
\hline$O(3 A)^{v} O(3 W)$ & & & $2.730(8)$ & \\
\hline $\mathrm{O}(2 \mathrm{~A})^{\mathrm{v}} \mathrm{O}(2 \mathrm{~W})$ & & & $2.791(4)$ & \\
\hline $\mathrm{O}(2 \mathrm{~B})^{\mathrm{vi}} \mathrm{O}(1 \mathrm{~W})$ & & & $2.876(4)$ & \\
\hline
\end{tabular}

Symmetry code: (i) $1-x, 1-y, 1-z$ (ii) $x, 1 / 2-y,-1 / 2+z$ (iii) $x, 3 / 2-y$, $1 / 2+z$ (iv) $-x, 1-y, 1-z$ (v) $1-x,-1 / 2+y, 1 / 2-z$ (vi) $-x, 1 / 2+y, 3 / 2-z$

005-D00006) is gratefully acknowledged. A single-crystal Xray analysis was performed using a Bruker SMART CCD diffractometer at the Central Laboratory, Gyeongsang National University.

\section{References}

1. J. Blaser, B. B. Stone, M. C. Groner, and S. H. Zinner, Antimicrob. Agents Chemother., 1987, 31, 1054.

2. P. Ball, J. Antimicrob. Chemother., 2000, 46, Topic T1, 17.

3. Internet homepage of MEDLINEplus, Health Information, A service of the National Library of Medicine / National Institute of Health, http://www.nlm.nih.gov/medlineplus/. 